OPEN ACCESS

Edited by:

Shanchun Guo,

Xavier University of Louisiana,

United States

Reviewed by:

Yongzhen Huang,

Northwest A\&F University, China

Yutian Zou,

Sun Yat-sen University Cancer Center

(SYSUCC), China

*Correspondence:

Renhua Wan

zww726696@sina.com

${ }^{t}$ These authors have contributed

equally to this work

Specialty section:

This article was submitted to

Molecular Medicine

a section of the journal

Frontiers in Cell and Developmental

Biology

Received: 19 September 2020

Accepted: 13 November 2020

Published: 01 December 2020

Citation:

Jiang $C$, Zeng $X$, Shan $R$, Wen $W$ Li J, Tan J, Li L and Wan R (2020) The Emerging Picture of the Roles of CircRNA-CDR1as in Cancer. Front. Cell Dev. Biol. 8:590478. doi: $10.3389 /$ fcell.2020.590478

\section{The Emerging Picture of the Roles of CircRNA-CDR1as in Cancer}

\author{
Chaohua Jiang ${ }^{1+}$, Xiaohong Zeng ${ }^{2 t}$, Renfeng Shan' ${ }^{1}$ Wu Wen ${ }^{1}$, Jianfeng Li', \\ Jinfeng Tan'1, Lei Li' ${ }^{1}$ and Renhua Wan ${ }^{1 *}$
}

${ }^{1}$ Department of General Surgery, The First Affiliated Hospital of Nanchang University, Nanchang University, Nanchang, China, ${ }^{2}$ Imaging Department, The First Affiliated Hospital of Nanchang University, Nanchang University, Nanchang, China

Circular RNAs (circRNAs) are covalently closed circular structures without $5^{\prime}$ caps and $3^{\prime}$ tails, which are mainly formed from precursor mRNAs (pre-mRNAs) via backsplicing of exons. With the development of RNA sequencing and bioinformatic analysis, circRNAs were recently rediscovered and found to be widely expressed in the tree of life. Cerebellar degeneration-related protein 1 antisense RNA (CDR1as) is recognized as one of the most well-identified circRNAs. It contains over 70 miR-7 binding sites and can regulate gene activity by sponging miR-7. Increasing numbers of studies have recently demonstrated that CDR1as is abnormally expressed in many types of tumors, such as colorectal cancer, cholangiocarcinoma and osteosarcoma, and plays a vital role in the development of cancer. However, there are few reviews focusing on CDR1as and cancer. Hence, it is important to review and discuss the role of CDR1as in cancer. Here, we first review the main biological features of CDR1as. We then focus on the expression and roles of CDR1as in cancer. Finally, we summarize what is known on the role of CDR1as in cancer and discuss future prospects in this area of research.

Keywords: circular RNA, CDR1as, cancer, biomarker, miRNA sponge

\section{INTRODUCTION}

Circular RNAs (circRNAs) are covalently closed circular structures without $5^{\prime}$ caps and $3^{\prime}$ tails, which are mainly formed from precursor mRNAs (pre-mRNAs) via the back-splicing of exons (Hansen et al., 2013a; Jeck et al., 2013; Memczak et al., 2013; Jeck and Sharpless, 2014; Lasda and Parker, 2014). The electron-microscopic discovery of circular RNAs in an RNA virus was first reported in Sanger et al. (1976). With the development of RNA sequencing and bioinformatic analysis, circRNAs were recently rediscovered and found to be widely expressed in the tree of life (Wang et al., 2010, 2014; Salzman et al., 2013). Furthermore, the expression of circular RNAs is cell type- and tissue-specific (Barrett and Salzman, 2016; Qian et al., 2020; Zhang S. et al., 2020), implying that the expression of circular RNAs is influenced by the specific cellular environment. As circRNAs lack $3^{\prime}$ or $5^{\prime}$ tails, they are more resistant to degradation by exonuclease RNase $\mathrm{R}$ and have longer half-lives than associated linear mRNAs (Chen, 2016; Patop and Kadener, 2018; Shang et al., 2019), which indicated that circRNAs could be more easily detectable biomarkers.

CircRNAs exert their function through several mechanisms (Geng et al., 2018; Patop and Kadener, 2018; Zhou et al., 2018; Arnaiz et al., 2019). Firstly, circRNAs can function as miRNA 
sponges and competitive endogenous RNAs to modulate the activity of miRNAs by competing for miRNA-binding sites (Salmena et al., 2011; Hansen et al., 2013a; Rong et al., 2017; Dori and Bicciato, 2019; Verduci et al., 2019; Lin et al., 2020; Liang et al., 2020). Secondly, some circRNAs can bind and interact with transcription factors to regulate the transcription of targeted gene (Memczak et al., 2013; Braunschweig et al., 2014). For example, ci-Ankyrin Repeat Domain 52 (ci-ANKRD52) can bind to the Pol II elongation complex and in result the accumulation of elongation Pol II at transcriptional sites to promote the transcription of ANKRD52 (Zhang et al., 2013). Thirdly, circRNAs can bind to target proteins to influence their decay or accumulation (Schneider et al., 2016; Abdelmohsen et al., 2017). For example, circ-Foxo3 can promote MDM2induced p53 ubiquitination by binding to both NDM2 and p53 protein (Du et al., 2017).

Cerebellar degeneration-related protein 1 antisense RNA (CDR1as), also known as circular RNA sponge for miR-7 (ciRS7) (Memczak et al., 2013), is recognized as one of the most well-identified circRNAs (Hansen et al., 2011, 2013a). As there are over $70 \mathrm{miR}-7$ binding sites in CDRlas, it acts as a miR7 sponge and regulates the latter's activity (Hansen et al., 2011, 2013a; Yao et al., 2018). Additionally, CDRlas is globally coexpressed with miR-7 in the brain, indicating that ciRS-7 may be a better term for it. However, none of the 70 identified binding sites of miR-7 in CDRlas are complementary with the entire miR-7 sequence, and only match the 5 end "seed region" of miR7 (Guo et al., 2020). Recently, there is increasing evidence that CDRlas is overexpressed in many tumor types, such as colorectal cancer (Tang et al., 2017), cholangiocarcinoma (Jiang et al., 2018) and osteosarcoma (Xu et al., 2018), and plays a vital role in the development of cancer. However, there are few reviews focusing on CDRlas and cancer. Hence, it is important to review and discuss the role of CDR1as in cancer.

\section{THE BIOGENESIS OF CDR1AS}

Hansen et al. (2011) found that miR-671 could decrease the levels of CDR1 mRNA by inducing the cleavage of the natural antisense transcript (NAT) of CDR1 mediated by argonaute (AGO)-2. They used 3 Rapid amplification of cDNA ends (RACE) analyses to characterize it and failed to find any polyadenylation, which implied that there is no poly(A)-tail in CDR1 NAT (Hansen et al., 2011). Furthermore, CDR1 NAT was found to be resistant to nicotinamide pyrophosphatase and terminal 5-phosphatedependent exonuclease, implying that there is also no $5^{\prime}$-terminal cap in the natural antisense transcript (NAT) of CDR1 (Hansen et al., 2011). Taken together, these results indicated that the natural antisense transcript (NAT) of CDR1 is a circular RNA. Generally, the formation of circular RNAs is often facilitated by flanking inverted repeats of the primate-specific Alu elements (Chen, 2016; Li et al., 2018a). However, the gene locus of CDRlas lacks Alu elements, indicating that there must be another mechanism of CDR1 NAT circularization. Barrett et al. found the promoter of LINC00632 could promote the expression of CDRlas, and the LINC00632 locus contains the CDR1as sequence (Barrett et al., 2017). According to their research, it stands to reason that the pre-linear RNA of CDR1as transcribed by the antisense strand of CDR1 concomitantly undergoes back-splicing of the $5^{\prime}$ and $3^{\prime}$ ends to form the circular RNA (Barrett et al., 2017; Figure 1).

\section{THE BIOLOGICAL FUNCTIONS OF CDR1AS}

CDRlas is a closed circular RNA formed from the antisense transcript of the cerebellum degeneration-related antigen 1 (CDR1) gene, whose length is 1,485 bp (Hansen et al., 2013b). CDRlas is highly expressed in the human brain and plays a vital role in midbrain development (Memczak et al., 2013), which might account for its relationship with neurodegenerative diseases, such as Alzheimer and Parkinson's disease (Shi et al., 2017; Azari et al., 2020). And the overexpression of CDR1as in zebrafish resulted in defects in the midbrain region similar to the knockdown of miR-7 (Memczak et al., 2013). At the same time, CDRlas was found to regulate the transcription and secretion of insulin by sponging miR-7 in pancreatic islet cells (Xu et al., 2015), which might be a potential therapeutic target for diabetes. As CDRlas can regulate the activity of miR-7 by sponging it, CDRlas exerts a wide range of physiological and pathological effects (Li et al., 2018b). Accordingly, CDRlas plays an important role in cellular proliferation and differentiation as well as cancer invasion and metastasis (Yan et al., 2018; Li L. et al., 2019; Wang et al., 2019; Yang L. et al., 2019; Zhou et al., 2019; Zou et al., 2019; Chen et al., 2020; Hanniford et al., 2020; Yang et al., 2020; Zhao F. et al., 2020).

\section{CDR1AS FUNCTIONS AS A MIRNA SPONGE}

There is increasing evidence that CDRlas can act as an miRNA sponge by absorbing several miRNAs (Shi et al., 2017; Kyei et al., 2020). Among these miRNAs, miR-7 functions as a tumor suppressor in many cancer types, such as osteosarcoma, breast cancer, hepatocellular carcinoma, and colorectal cancer (Yu et al., 2016; Tang et al., 2017; Yang X. et al., 2017; Xu et al., 2018). CDRlas was found to promote the proliferation and metastasis of cancer cells by sponging miR-7 (Tang et al., 2017; Xu et al., 2018; Yang L. et al., 2019). Furthermore, since conserved miR7 target sites on CDRlas are complementary to miR-7, these sites could be a lodging site for transport (Memczak et al., 2013; Piwecka et al., 2017). Furthermore, CDRlas was able to sponge miR-1270 and modulate the activity of miR-1270, resulting in drug resistance, proliferation and metastasis of cancer (Su et al., 2019; Yuan et al., 2019; Zhao et al., 2019). Similarly, CDR1as was found to promote the progression of cholangiocarcinoma and osteoarthritis by sponging miR-641 (Li D. et al., 2020; Zhang W. et al., 2020). Moreover, CDR1as was reported to sponge miR-135b-5p, miR-219a, miR-1299, and miR-876-5p in ovarian cancer, non-small-cell lung cancer and esophageal squamous cell carcinoma, respectively (Sang et al., 2018b; Chen et al., 2019; 


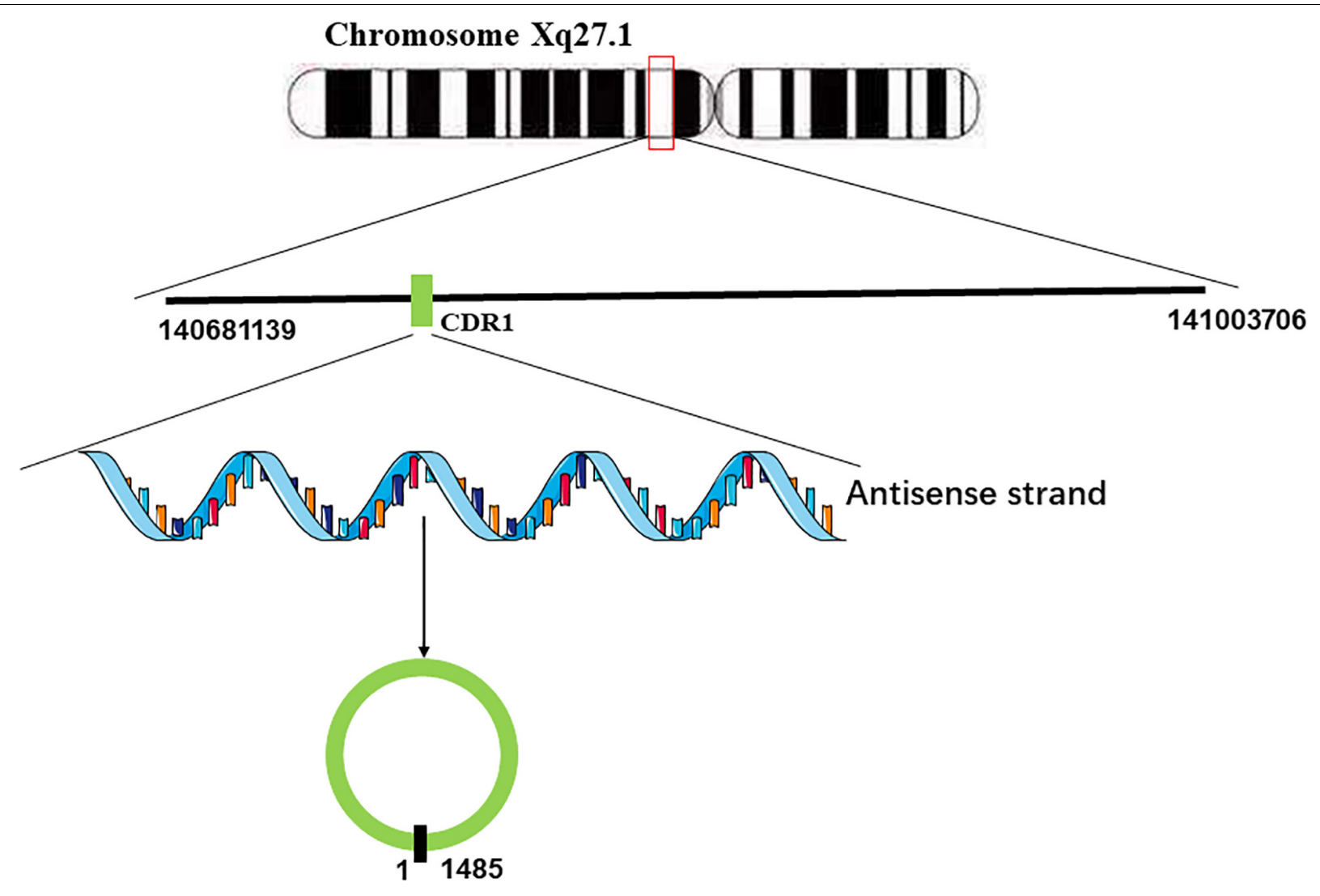

FIGURE 1 | Generation of CDR1as. CDR1 is located on Chromosome Xq27.1. The antisense strand of CDR1 undergoes back-splicing of the $5^{\prime}$ and $3^{\prime}$ ends to form the circular RNA.

Cai et al., 2020; Li Y. et al., 2020; Meng et al., 2020). In addition, CDRlas was found to stimulate tube formation in microvascular endothelial cells by decreasing the expression of miR-26a-5p (Cui et al., 2020). Taken together, these studies demonstrate that CDR1as plays varied roles in the occurrence and development of cancer and might be a potential therapeutic target.

\section{THE EXPRESSION OF CDR1AS IN CANCER}

Many studies have demonstrated that CDRlas is expressed abnormally in many cancer types (Pan et al., 2018; Su et al., 2018; Tanaka et al., 2019; Lin et al., 2020; Tian et al., 2020; Zhou et al., 2020). The expression of CDR1as is upregulated in most tumors, such as colorectal cancer, hepatocellular carcinoma and breast cancer, and it exerts a tumor-promoting effect (Tang et al., 2017; Weng et al., 2017; Xu et al., 2017; Yang X. et al., 2017; Yuan et al., 2019; Zhang Z. et al., 2020; Zhao Y. H. et al., 2020). Especially in colon cancer, Kristensen et al. (2020) found that CDR1as is absent in the cancer cells, but highly expressed in stromal cells within the tumor microenvironment. Similarly, CDRlas showed low expression in other tumors, such as ovarian cancer, melanoma and bladder cancer, indicating that it functions as a tumor suppressor (Chen et al., 2019; Zhao et al., 2019). These findings indicate that CDRlas can function either as a tumor suppressor or promoter in different tumor microenvironments, which will be discussed below (Table 1).

\section{THE ROLES OF CDR1AS IN CANCER}

\section{The Roles of CDR1as in Tumor Growth}

Numerous studies have demonstrated that CDR1as is involved in the regulation of tumor growth by sponging several miRNAs and regulating multiple signaling pathways. For example, miR-7 acts as a tumor suppressor that can regulate cellular proliferation and various biological process by triggering the signal transduction of the growth factors (Sun et al., 2016; Yang Z. et al., 2017). CDR1as is upregulated in numerous cancers, where it can sponge miR-7 and stimulate the expression of the downstream targeted genes, such as E2F3, EGFR, IGF-1R, CCNE1, PIK3CD, RAF1, PTEN, and KLF4. Accordingly, CDR1as was found to promote tumor proliferation in nasopharyngeal carcinoma, colorectal cancer, non-small-cell lung cancer, osteosarcoma, laryngeal squamous cell carcinoma, gastric cancer and esophageal squamous cell carcinoma, respectively (Tang et al., 2017; Yang X. et al., 2017; Li R. C. et al., 2018; Pan et al., 2018; Xu et al., 2018; Zhang J. et al., 2018; Zhang X. et al., 2018; Huang et al., 2019; Zhong et al., 2019; Yang et al., 2020). These studies demonstrated 
TABLE 1 | The expression and roles of circ-CDR1as in different human cancers.

\begin{tabular}{|c|c|c|c|c|}
\hline Cancer type & Expression & Functional roles & Related signaling pathways & References \\
\hline Melanoma & Downregulated & Migration & CDR1as-IGF2BP3 & $\begin{array}{l}\text { Sun et al., 2016; Zhang L. et al., 2018; } \\
\text { Hanniford et al., } 2020\end{array}$ \\
\hline Non-small-cell lung cancer & Upregulated & $\begin{array}{l}\text { Proliferation } \\
\text { Migration } \\
\text { Apoptosis }\end{array}$ & CDR1as-219a-5p/SOX5 & Zhang X. et al., 2018; Li Y. et al., 2020 \\
\hline $\begin{array}{l}\text { Esophageal squamous cell } \\
\text { carcinoma }\end{array}$ & Upregulated & $\begin{array}{l}\text { Proliferation } \\
\text { Migration } \\
\text { Autophagy }\end{array}$ & $\begin{array}{l}\text { CDR1as-miR-1299-EGFR } \\
\text { CDR1as-miR-7-KLF4 } \\
\text { CDR1as-miR-7-HOXB13 } \\
\text { CDR1as-miR-876-5p-MAGE-A }\end{array}$ & $\begin{array}{l}\text { Li R. C. et al., 2018; Sang et al., 2018b; } \\
\text { Huang et al., 2019; Meng et al., } 2020\end{array}$ \\
\hline Colorectal cancer & Upregulated & $\begin{array}{l}\text { Proliferation } \\
\text { Migration }\end{array}$ & CDR1as-miR-7/HOXB13 & $\begin{array}{l}\text { Tang et al., 2017; Weng et al., 2017; } \\
\text { Li R. C. et al., } 2018\end{array}$ \\
\hline Hepatocellular carcinoma & Upregulated & $\begin{array}{l}\text { Proliferation } \\
\text { Migration }\end{array}$ & CDR1as-miR-7 & $\begin{array}{l}\text { Yu et al., 2016; Xu et al., 2017; } \\
\text { Yang X. et al., 2017; Su et al., } 2019\end{array}$ \\
\hline Nasopharyngeal carcinoma & Upregulated & Proliferation & CDR1as-miR-7-E2F3 & Zhong et al., 2019 \\
\hline $\begin{array}{l}\text { Laryngeal squamous cell } \\
\text { carcinoma }\end{array}$ & Upregulated & $\begin{array}{l}\text { Proliferation } \\
\text { Migration }\end{array}$ & $\begin{array}{l}\text { CDR1as-miR-7-CCNE1 } \\
\text { CDR1as-miR-7-PIK3CD }\end{array}$ & Zhang J. et al., 2018 \\
\hline Osteosarcoma & Upregulated & Proliferation & CDR1as-miR-7 & Xu et al., 2018 \\
\hline Cholangiocarcinoma & Upregulated & $\begin{array}{l}\text { Proliferation } \\
\text { Migration }\end{array}$ & CDR1as-miR-641 & Jiang et al., 2018; Li D. et al., 2020 \\
\hline Gastric cancer & Upregulated & $\begin{array}{l}\text { Proliferation } \\
\text { Migration } \\
\text { Chemoresistance }\end{array}$ & $\begin{array}{l}\text { CDR1as-miR-7-PTEN } \\
\text { CDR1as-miR-135-TRPC1 } \\
\text { CDR1as-miR-7-5p-REGgama }\end{array}$ & $\begin{array}{l}\text { Pan et al., 2018; Li C. et al., 2019; } \\
\text { Zhang Z. et al., } 2020\end{array}$ \\
\hline Breast cancer & Upregulated & $\begin{array}{l}\text { Proliferation } \\
\text { Migration } \\
\text { Chemoresistance }\end{array}$ & $\begin{array}{l}\text { CDR1as-miR-1299-EGFR } \\
\text { CDR1as-miR-7-REG } \gamma \\
\text { CDR1as-miR-1299-MMPs }\end{array}$ & $\begin{array}{l}\text { Sang et al., 2018a; Uhr et al., 2018; } \\
\text { Yang et al., 2019a,b; Meng et al., } 2020\end{array}$ \\
\hline Ovarian cancer & Downregulated & $\begin{array}{l}\text { Proliferation-inhibition } \\
\text { Migration-Inhibition } \\
\text { Chemosensitivity }\end{array}$ & $\begin{array}{l}\text { CDR1as-miR-135B-5P } \\
\text { CDR1as-miR-1270-SCAI }\end{array}$ & Chen et al., 2019; Zhao et al., 2019 \\
\hline Bladder cancer & Downregulated & $\begin{array}{l}\text { Proliferation-inhibition } \\
\text { Migration-Inhibition } \\
\text { Chemosensitivity }\end{array}$ & $\begin{array}{l}\text { CDR1as-miR1270-APAF1 } \\
\text { CDR1as-miR-135a }\end{array}$ & Li P. et al., 2018; Yuan et al., 2019 \\
\hline
\end{tabular}

that the CDR1as/miR-7 axis plays a vital role in tumor growth and might be a potential target for cancer therapy. AFP (alphafetoprotein), a biomarker of hepatocellular carcinoma, is the target gene of miR-1270. CDRlas can sponge miR-1270 and upregulate the expression of AFP to promote tumor growth, invasion and metastasis in hepatocellular carcinoma ( $\mathrm{Su}$ et al., 2019). Similarly, the overexpression of CDRlas can stimulate tumor proliferation, invasion and metastasis by sponging miR641 and stimulating the expression of AKT3 and mTOR in cholangiocarcinoma (Li D. et al., 2020). Furthermore, CDR1as knockdown inhibited tumor growth, invasion and metastasis by regulating the miR-219a-5p/SOX5 axis in non-small-cell lung cancer (Li Y. et al., 2020). Similarly, the knockdown of CDRlas could inhibit tumor growth via miR-135p in ovarian cancer (Chen et al., 2019). However, CDRlas was reported to inhibit tumor growth in glioblastoma multiforme by disrupting p53/MDM2 complex formation (Lou et al., 2020), which implies that CDR1as has different effects on cell growth in different cancer types (Figure 2).

\section{The Roles of CDR1as in Cancer Metastasis}

Cancer metastasis is the critical step of cancer development and progression and many studies reported that CDR1as could influence cancer metastasis. As described above, CDR1as can promote cancer metastasis via multiple signaling pathways (Su et al., 2019; Li D. et al., 2020; Li Y. et al., 2020). In addition, CDR1as was found to interact with IGF2BP3 to promote tumor invasion and metastasis in melanoma (Zhang $\mathrm{L}$. et al., 2018; Hanniford et al., 2020). Similarly, CDR1as could enhance the expression of MAGE-A family by sponging miR876-5p to promote the progression of esophageal squamous cell carcinoma (Sang et al., 2018b). Furthermore, CDR1as could regulate miR-7/HOXB13 and miR-1299/MMPs to promote the metastasis of esophageal squamous cell carcinoma and triple-negative breast cancer, respectively ( $\mathrm{Li}$ R. C. et al., 2018; Sang et al., 2018a). However, CDRlas was found to sponge miR-135b-5p and upregulate the expression of HIF1AN to inhibit the growth, invasion and metastasis of ovarian cancer (Chen et al., 2019), which shows that the roles of CDRlas in tumor metastasis vary in different cacer types (Figure 2).

\section{The Roles of CDR1as in Cancer Chemoresistance}

Chemoresistance is the main obstacle to cancer therapy and remains a great challenge for improving the clinical outcomes of cancer patients (Zheng, 2017; Yeldag and Rice, 


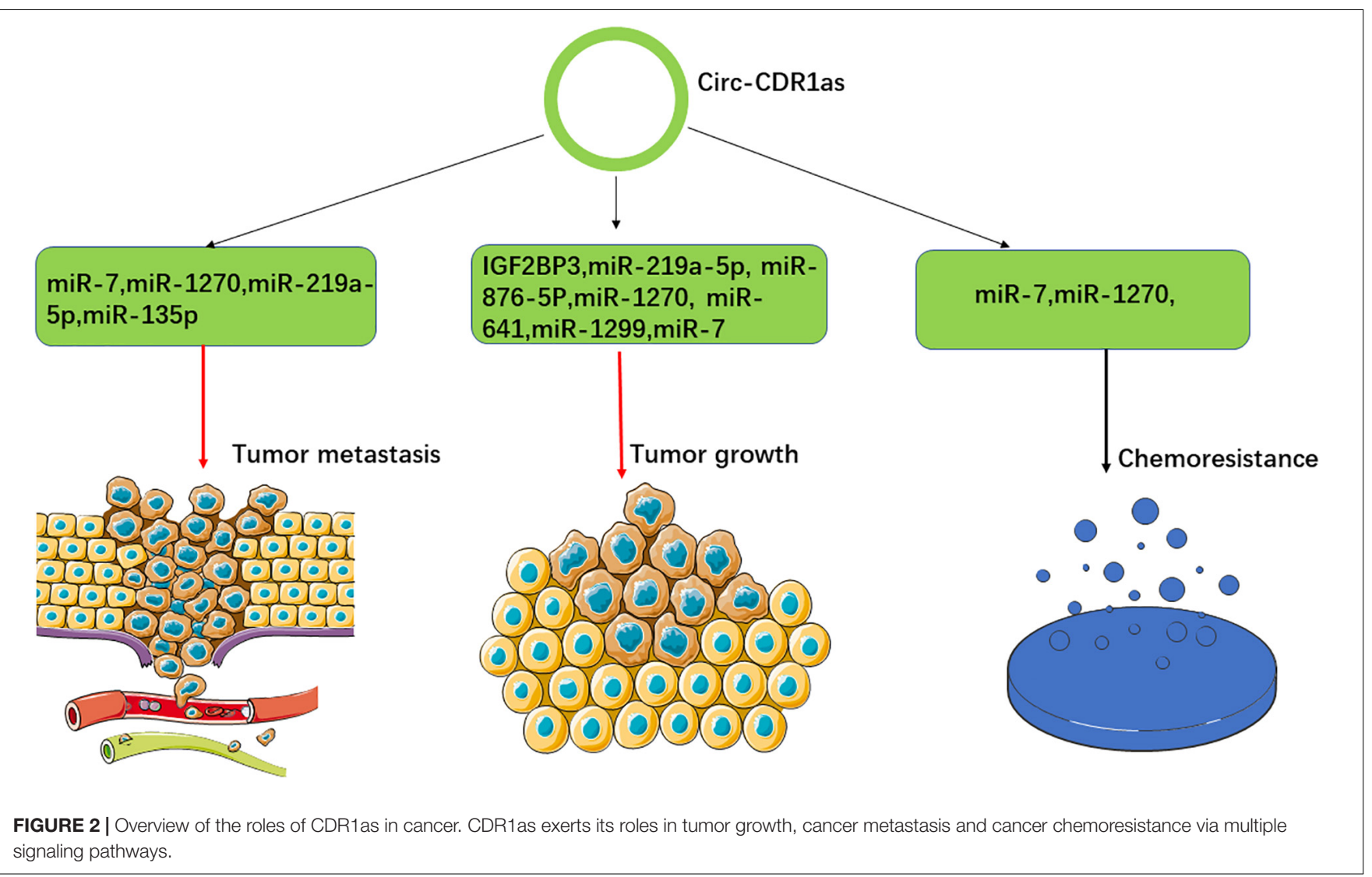

2018). A number of studies have demonstrated that the dysregulated expression of CDRlas is related to cancer chemoresistance (Uhr et al., 2018; Yang et al., 2019a,b; Mao and $\mathrm{Xu}, 2020$ ). Two studies showed that the knockdown of CDRlas could increase the chemosensitivity of 5-fluorouracyland cisplatin-resistant breast cancer cells by sponging miR-7 (Yang et al., 2019a,b). Similarly, downregulation of CDR1as could modulate the miR-7-5p/REG $\gamma$ axis to promote low-dose diosbulbin-B-induced gastric cancer cell death (Li C. et al., 2019). Furthermore, another study reported that CDR1as could regulate stemness and promote cisplatin chemoresistance in NSCLC cells by targeting the miR-641/HOXA9 axis (Zhao Y. et al., 2020). Taken together, these results indicate that CDRlas might be a potential therapeutic target for overcoming cancer chemoresistance. However, other studies demonstrated that the overexpression of CDRlas could increase the sensibility to cisplatin by sponging miR-1270 in bladder and ovarian cancer (Yuan et al., 2019; Zhao et al., 2019). The inconsistent results demonstrate that the roles of CDRlas in chemoresistance vary in different cancer types (Figure 2).

\section{CONCLUSION AND PERSPECTIVES}

CircRNAs were previously considered to be the products of faulty RNA splicing (Kristensen et al., 2018). However, with the development of RNA sequencing and bioinformatic analysis, circRNAs were recently rediscovered and found to be widely expressed in the tree of life (Wang et al., 2010, 2014; Salzman et al., 2013). Increasing numbers of studies demonstrated that circRNAs are abnormally expressed in cancer and exert a vital role in cancer progression through a complicated gene regulatory network. Here, we firstly systematically reviewed and discussed the roles of CDRlas in cancer, listing studies that might deepen our understanding of how it modulates cancer progression. Additionally, the relationship between CDRlas expression and clinicopathological characteristics was summarized. In detail, high CDR1as expression was associated with worse clinicopathological characteristics, including the $\mathrm{T}$ status, $\mathrm{N}$ status, histological grade, TNM stage and distant metastasis in solid tumors, such as esophageal squamous cell carcinoma (ESCC), non-small cell lung cancer (NSCLC), colorectal cancer (CC), and hepatocellular carcinoma (Zou et al., 2020). Numerous experiments have demonstrated that CDR1as might be an oncogene and promote cellular proliferation and cancer metastasis. In addition, the expression of CDR1as was found to be associated with poor prognosis in cancer patients. As circRNAs lack $3^{\prime}$ tails or $5^{\prime}$ caps, they are more resistant to degradation by exonuclease RNase $\mathrm{R}$ and have more long half-lives than associated linear mRNAs (Chen, 2016). Meantime, some studies reported that stably existed in human body fluids, such as serum, plasma, and saliva. Furthermore, CDR1as was reported to have a specificity of $74 \%$ in the diagnosis of digestive 
system-derived tumors (Zou et al., 2020). The AUC of the ROC curve represents the comprehensive accuracy rate of detection, and according to the results of this study, detection of CDR1as had an AUC of 0.84 (95\% CI, 0.80-0.87) in solid tumors (Zou et al., 2020). Taken together, these findings indicate that CDR1as might become an easily detectable prognostic factor for cancer patients. However, our knowledge on the roles of CDRlas in cancer is still limited and further studies are needed. The available reports mainly focused on the function of CDR1as as a miRNA sponge, but circRNAs can also bind to proteins to exert their function. Hence, the other possible functions of CDR1as in cancer should also be investigated. Importantly, various studies

\section{REFERENCES}

Abdelmohsen, K., Panda, A. C., Munk, R., Grammatikakis, I., Dudekula, D. B., De, S., et al. (2017). Identification of HuR target circular RNAs uncovers suppression of PABPN1 translation by CircPABPN1. RNA Biol. 14, 361-369. doi: 10.1080/15476286.2017.1279788

Arnaiz, E., Sole, C., Manterola, L., Iparraguirre, L., Otaegui, D., and Lawrie, C. H. (2019). CircRNAs and cancer: Biomarkers and master regulators. Semin. Cancer Biol. 58, 90-99. doi: 10.1016/j.semcancer.2018.12.002

Azari, H., Mousavi, P., Karimi, E., Sadri, F., Zarei, M., Rafat, M., et al. (2020). The expanding role of CDR1-AS in the regulation and development of cancer and human diseases. J. Cell Physiol. 2020, 1-20. doi: 10.1002/jcp.29950

Barrett, S. P., and Salzman, J. (2016). Circular RNAs: analysis, expression and potential functions. Development 143, 1838-1847. doi: 10.1242/dev.128074

Barrett, S. P., Parker, K. R., Horn, C., Mata, M., and Salzman, J. (2017). ciRS-7 exonic sequence is embedded in a long non-coding RNA locus. PLoS Genet. 13:e1007114. doi: 10.1371/journal.pgen.1007114

Braunschweig, U., Barbosa-Morais, N. L., Pan, Q., Nachman, E. N., Alipanahi, B., Gonatopoulos-Pournatzis, T., et al. (2014). Widespread intron retention in mammals functionally tunes transcriptomes. Gen. Res. 24, 1774-1786. doi: 10.1101 /gr.177790.114

Cai, W., Zhang, Y., and Su, Z. (2020). ciRS-7 targeting miR-135a-5p promotes neuropathic pain in CCI rats via inflammation and autophagy. Gene 736:144386. doi: 10.1016/j.gene.2020.144386

Chen, G., Wang, Q., Li, Z., Yang, Q., Liu, Y., Du, Z., et al. (2020). Circular RNA CDRlas promotes adipogenic and suppresses osteogenic differentiation of BMSCs in steroid-induced osteonecrosis of the femoral head. Bone 133:115258. doi: 10.1016/j.bone.2020.115258

Chen, H., Mao, M., Jiang, J., Zhu, D., and Li, P. (2019). Circular RNA CDRlas acts as a sponge of miR-135b-5p to suppress ovarian cancer progression. Onco Targets Ther. 12, 3869-3879. doi: 10.2147/OTT.S207938

Chen, L. L. (2016). The biogenesis and emerging roles of circular RNAs. Nat. Rev. Mol. Cell Biol. 17, 205-211. doi: 10.1038/nrm.2015.32

Cui, M., Shen, W., Qin, W., Wang, X., Li, Y., Xu, F., et al. (2020). Circular RNA ciRS-7 promotes tube formation in microvascular endothelial cells through downregulation of miR-26a-5p. J. Biochem. Mol. Toxicol 34:e22468. doi: 10. $1002 /$ jbt. 22468

Dori, M., and Bicciato, S. (2019). Integration of Bioinformatic Predictions and Experimental Data to Identify circRNA-miRNA Associations. Genes 10:642. doi: 10.3390/genes10090642

Du, W. W., Fang, L., Yang, W., Wu, N., Awan, F. M., Yang, Z., et al. (2017). Induction of tumor apoptosis through a circular RNA enhancing Foxo3 activity. Cell Death Differ. 24, 357-370. doi: 10.1038/cdd.2016.133

Geng, Y., Jiang, J., and Wu, C. (2018). Function and clinical significance of circRNAs in solid tumors. J. Hematol. Oncol. 11:98. doi: 10.1186/s13045-0180643-z

Guo, Z., Cao, Q., Zhao, Z., and Song, C. (2020). Biogenesis, Features, Functions, and Disease Relationships of a Specific Circular RNA: CDRlas. Aging Dis. 11, 1009-1020. doi: 10.14336/AD.2019.0920

Hanniford, D., Ulloa-Morales, A., Karz, A., Berzoti-Coelho, M. G., Moubarak, R. S., Sanchez-Sendra, B., et al. (2020). Epigenetic Silencing of CDR1as Drives showed that CDR1as exerts a vital role in cancer development, and we hope that therapies targeting CDR1as could be applied in the clinical treatment of cancer patients.

\section{AUTHOR CONTRIBUTIONS}

RS, WW, and JL collected the related manuscript. CJ and XZ drafted and revised the manuscript. RW designed the review. JT and LL participated in the design of the review and helped to draft and revise the manuscript. All authors read and approved the final manuscript.

IGF2BP3-Mediated Melanoma Invasion and Metastasis. Cancer Cell 37, 5570e15. doi: 10.1016/j.ccell.2019.12.007

Hansen, T. B., Jensen, T. I., Clausen, B. H., Bramsen, J. B., Finsen, B., Damgaard, C. K., et al. (2013a). Natural RNA circles function as efficient microRNA sponges. Nature 495, 384-388. doi: 10.1038/nature11993

Hansen, T. B., Kjems, J., and Damgaard, C. K. (2013b). Circular RNA and miR-7 in cancer. Cancer Res. 73, 5609-5612. doi: 10.1158/0008-5472.CAN-13-1568

Hansen, T. B., Wiklund, Bramsen, J. B., Villadsen, S. B., Statham, A. L., Clark, S. J., et al. (2011). miRNA-dependent gene silencing involving Ago2-mediated cleavage of a circular antisense RNA. EMBO J. 30, 4414-4422. doi: 10.1038/ emboj.2011.359

Huang, H., Wei, L., Qin, T., Yang, N., Li, Z., and Xu, Z. (2019). Circular RNA ciRS7 triggers the migration and invasion of esophageal squamous cell carcinoma via miR-7/KLF4 and NF-kappaB signals. Cancer Biol. Ther. 20, 73-80. doi: $10.1080 / 15384047.2018 .1507254$

Jeck, W. R., and Sharpless, N. E. (2014). Detecting and characterizing circular RNAs. Nat. Biotechnol. 32, 453-461. doi: 10.1038/nbt.2890

Jeck, W. R., Sorrentino, J. A., Wang, K., Slevin, M. K., Burd, C. E., Liu, J., et al. (2013). Circular RNAs are abundant, conserved, and associated with ALU repeats. Rna 19, 141-157. doi: 10.1261/rna.035667.112

Jiang, X. M., Li, Z. L., Li, J. L., Xu, Y., Leng, K. M., Cui, Y. F., et al. (2018). A novel prognostic biomarker for cholangiocarcinoma: circRNA Cdr1as. Eur. Rev. Med. Pharmacol.Sci. 22, 365-371. doi: 10.26355/eurrev_201801_14182

Kristensen, L. S., Ebbesen, K. K., Sokol, M., Jakobsen, T., Korsgaard, U., Eriksen, A. C., et al. (2020). Spatial expression analyses of the putative oncogene ciRS7 in cancer reshape the microRNA sponge theory. Nat. Commun. 11:4551. doi: 10.1038/s41467-020-18355-2

Kristensen, L. S., Hansen, T. B., Veno, M. T., and Kjems, J. (2018). Circular RNAs in cancer: opportunities and challenges in the field. Oncogene 37, 555-565. doi: 10.1038/onc.2017.361

Kyei, B., Li, L., Yang, L., Zhan, S., and Zhang, H. (2020). CDRlas/miRNAs-related regulatory mechanisms in muscle development and diseases. Gene 730:144315. doi: 10.1016/j.gene.2019.144315

Lasda, E., and Parker, R. (2014). Circular RNAs: diversity of form and function. Rna 20, 1829-1842. doi: 10.1261/rna.047126.114

Li, C., Li, M., and Xue, Y. (2019). Downregulation of CircRNA CDRlas specifically triggered low-dose Diosbulbin-B induced gastric cancer cell death by regulating miR-7-5p/REGgamma axis. Biomed. Pharmacother. 120:109462. doi: 10.1016/j. biopha.2019.109462

Li, D., Tang, Z., Gao, Z., Shen, P., Liu, Z., and Dang, X. (2020). Circular RNA CDRlas exerts oncogenic properties partially through regulating miR-641 in cholangiocarcinoma. Mol. Cell Biol. 40, e42-e20. doi: 10.1128/MCB.00042-20

Li, L., Chen, Y., Nie, L., Ding, X., Zhang, X., Zhao, W., et al. (2019). MyoDinduced circular RNA CDR1as promotes myogenic differentiation of skeletal muscle satellite cells. Biochim. Biophys. Acta Gene Regul. Mecha. 1862, 807-821. doi: 10.1016/j.bbagrm.2019.07.001

Li, P., Yang, X., Yuan, W., Yang, C., Zhang, X., Han, J., et al. (2018). CircRNACdrlas Exerts Anti-Oncogenic Functions in Bladder Cancer by Sponging MicroRNA-135a. Cell Physiol. Biochem. 46, 1606-1616. doi: 10.1159/000489208

Li, R. C., Ke, S., Meng, F. K., Lu, J., Zou, X. J., He, Z. G., et al. (2018). CiRS-7 promotes growth and metastasis of esophageal squamous cell carcinoma via 
regulation of miR-7/HOXB13. Cell Death Dis. 9:838. doi: 10.1038/s41419-018$0852-y$

Li, X., Yang, L., and Chen, L. L. (2018a). The Biogenesis, Functions, and Challenges of Circular RNAs. Mol. Cell 71, 428-442. doi: 10.1016/j.molcel.2018.06.034

Li, X., Zheng, Y., Zheng, Y., Huang, Y., Zhang, Y., Jia, L., et al. (2018b). Circular RNA CDRlas regulates osteoblastic differentiation of periodontal ligament stem cells via the miR-7/GDF5/SMAD and p38 MAPK signaling pathway. Stem Cell Res. Ther. 9:232. doi: 10.1186/s13287-018-0976-0

Li, Y., Zhang, J., Pan, S., Zhou, J., Diao, X., and Liu, S. (2020). CircRNA CDR1as knockdown inhibits progression of non-small-cell lung cancer by regulating miR-219a-5p/SOX5 axis. Thorac Cancer 11, 537-548. doi: 10.1111/1759-7714. 13274

Liang, Z. Z., Guo, C., Zou, M. M., Meng, P., and Zhang, T. T. (2020). circRNAmiRNA-mRNA regulatory network in human lung cancer: an update. Cancer Cell. Int. 20:173. doi: 10.1186/s12935-020-01245-4

Lin, F., Chen, H. W., Zhao, G. A., Li, Y., He, X. H., Liang, W. Q., et al. (2020). Advances in Research on the circRNA-miRNA-mRNA Network in Coronary Heart Disease Treated with Traditional Chinese Medicine. Evid Based Complement Alternat. Med. 2020:8048691. doi: 10.1155/2020/8048691

Lou, J., Hao, Y., Lin, K., Lyu, Y., Chen, M., Wang, H., et al. (2020). Circular RNA CDR1as disrupts the p53/MDM2 complex to inhibit Gliomagenesis. Mol. Cancer 19:138. doi: 10.1186/s12943-020-01253-y

Mao, Y., and Xu, R. (2020). Circular RNA CDR1-AS contributes to pemetrexed and cisplatin chemoresistance through EGFR/PI3K signaling pathway in lung adenocarcinoma. Biomed. Pharmacother. 123:109771. doi: 10.1016/j.biopha. 2019.109771

Memczak, S., Jens, M., Elefsinioti, A., Torti, F., Krueger, J., Rybak, A., et al. (2013). Circular RNAs are a large class of animal RNAs with regulatory potency. Nature 495, 333-338. doi: 10.1038/nature11928

Meng, L., Liu, S., Ding, P., Chang, S., and Sang, M. (2020). Circular RNA ciRS-7 inhibits autophagy of ESCC cells by functioning as miR-1299 sponge to target EGFR signaling. J. Cell Biochem. 121, 1039-1049. doi: 10.1002/jcb.29339

Pan, H., Li, T., Jiang, Y., Pan, C., Ding, Y., Huang, Z., et al. (2018). Overexpression of Circular RNA ciRS-7 Abrogates the Tumor Suppressive Effect of miR-7 on Gastric Cancer via PTEN/PI3K/AKT Signaling Pathway. J. Cell Biochem. 119, 440-446. doi: 10.1002/jcb.26201

Patop, I. L., and Kadener, S. (2018). circRNAs in Cancer. Curr. Opin. Genet. Dev. 48, 121-127. doi: 10.1016/j.gde.2017.11.007

Piwecka, M., Glazar, P., Hernandez-Miranda, L. R., Memczak, S., Wolf, S. A., Rybak-Wolf, A., et al. (2017). Loss of a mammalian circular RNA locus causes miRNA deregulation and affects brain function. Science 357, 6357. doi: 10.1126/ science.aam8526

Qian, Y., Chopp, M., and Chen, J. (2020). Emerging role of microRNAs in ischemic stroke with comorbidities. Exp. Neurol. 331, 113382. doi: 10.1016/j.expneurol. 2020.113382

Rong, D., Sun, H., Li, Z., Liu, S., Dong, C., Fu, K., et al. (2017). An emerging function of circRNA-miRNAs-mRNA axis in human diseases. Oncotarget 8, 73271-73281. doi: 10.18632/oncotarget.19154

Salmena, L., Poliseno, L., Tay, Y., Kats, L., and Pandolfi, P. P. A. (2011). ceRNA hypothesis: the Rosetta Stone of a hidden RNA language? Cell 146, 353-358. doi: 10.1016/j.cell.2011.07.014

Salzman, J., Chen, R. E., Olsen, M. N., Wang, P. L., and Brown, P. O. (2013). Cell-type specific features of circular RNA expression. PLoS Gen. 9:e1003777. doi: 10.1371 /journal.pgen.1003777

Sang, M., Meng, L., Liu, S., Ding, P., Chang, S., Ju, Y., et al. (2018a). Circular RNA ciRS-7 Maintains Metastatic Phenotypes as a ceRNA of miR-1299 to Target MMPs. Mol. Cancer Res. 16, 1665-1675. doi: 10.1158/1541-7786.MCR-18-0284

Sang, M., Meng, L., Sang, Y., Liu, S., Ding, P., Ju, Y., et al. (2018b). Circular RNA ciRS-7 accelerates ESCC progression through acting as a miR-876-5p sponge to enhance MAGE-A family expression. Cancer Lett. 426, 37-46. doi: 10.1016/j.canlet.2018.03.049

Sanger, H. L., Klotz, G., Riesner, D., Gross, H. J., and Kleinschmidt, A. K. (1976). Viroids are single-stranded covalently closed circular RNA molecules existing as highly base-paired rod-like structures. Proc. Natl. Acad. Sci. U S A. 73, 3852-3856. doi: 10.1073/pnas.73.11.3852

Schneider, T., Hung, L. H., Schreiner, S., Starke, S., Eckhof, H., Rossbach, O., et al. (2016). CircRNA-protein complexes: IMP3 protein component defines subfamily of circRNPs. Sci. Rep. 6:31313. doi: 10.1038/srep31313
Shang, Q., Yang, Z., Jia, R., and Ge, S. (2019). The novel roles of circRNAs in human cancer. Mol. Cancer 18, 6. doi: 10.1186/s12943-018-0934-6

Shi, Z., Chen, T., Yao, Q., Zheng, L., Zhang, Z., Wang, J., et al. (2017). The circular RNA ciRS-7 promotes APP and BACE1 degradation in an NF-kappaBdependent manner. FEBS J. 284, 1096-1109. doi: 10.1111/febs.14045

Su, C., Han, Y., Zhang, H., Li, Y., Yi, L., Wang, X., et al. (2018). CiRS-7 targeting miR-7 modulates the progression of non-small cell lung cancer in a manner dependent on NF-kappaB signalling. J. Cell Mol. Med. 22, 3097-3107. doi: 10.1111/jcmm.13587

Su, Y., Lv, X., Yin, W., Zhou, L., Hu, Y., Zhou, A., et al. (2019). CircRNA Cdr1as functions as a competitive endogenous RNA to promote hepatocellular carcinoma progression. Aging 11, 8182-8203. doi: 10.18632/aging.10 2312

Sun, X., Li, J., Sun, Y., Zhang, Y., Dong, L., Shen, C., et al. (2016). miR-7 reverses the resistance to BRAFi in melanoma by targeting EGFR/IGF-1R/CRAF and inhibiting the MAPK and PI3K/AKT signaling pathways. Oncotarget 7, 5355853570. doi: 10.18632/oncotarget.10669

Tanaka, E., Miyakawa, Y., Kishikawa, T., Seimiya, T., Iwata, T., Funato, K., et al. (2019). Expression of circular RNA CDR1AS in colon cancer cells increases cell surface PDL1 protein levels. Oncol. Rep. 42, 1459-1466. doi: 10.3892/or.2019. 7244

Tang, W., Ji, M., He, G., Yang, L., Niu, Z., Jian, M., et al. (2017). Silencing CDRlas inhibits colorectal cancer progression through regulating microRNA7. OncoTarg. Ther. 10, 2045-2056. doi: 10.2147/OTT.S131597

Tian, G., Li, G., Guan, L., Wang, Z., and Li, N. (2020). Prognostic Value of Circular RNA ciRS-7 in Various Cancers: A PRISMA-Compliant Meta-Analysis. Biomed. Res. Int. 2020:1487609. doi: 10.1155/2020/1487609

Uhr, K., Sieuwerts, A. M., de Weerd, V., Smid, M., Hammerl, D., Foekens, J. A., et al. (2018). Association of microRNA-7 and its binding partner CDR1-AS with the prognosis and prediction of 1(st)-line tamoxifen therapy in breast cancer. Sci. Rep. 8:9657. doi: 10.1038/s41598-018-27987-w

Verduci, L., Strano, S., Yarden, Y., and Blandino, G. (2019). The circRNAmicroRNA code: emerging implications for cancer diagnosis and treatment. Mol. Oncol. 13, 669-680. doi: 10.1002/1878-0261.12468

Wang, F., Chen, X., Han, Y., Xi, S., and Wu, G. (2019). circRNA CDR1as Regulated the Proliferation of Human Periodontal Ligament Stem Cells under a Lipopolysaccharide-Induced Inflammatory Condition. Med. Inflamm 2019:1625381. doi: 10.1155/2019/1625381

Wang, K., Singh, D., Zeng, Z., Coleman, S. J., Huang, Y., Savich, G. L., et al. (2010). MapSplice: accurate mapping of RNA-seq reads for splice junction discovery. Nucl. Acids Res.38:e178. doi: 10.1093/nar/gkq622

Wang, P. L., Bao, Y., Yee, M. C., Barrett, S. P., Hogan, G. J., Olsen, M. N., et al. (2014). Circular RNA is expressed across the eukaryotic tree of life. PLoS One 9:e90859. doi: 10.1371/journal.pone.0090859

Weng, W., Wei, Q., Toden, S., Yoshida, K., Nagasaka, T., Fujiwara, T., et al. (2017). Circular RNA ciRS-7-A Promising Prognostic Biomarker and a Potential Therapeutic Target in Colorectal Cancer. Clin. Cancer Res. 23, 3918-3928. doi: 10.1158/1078-0432.CCR-16-2541

Xu, B., Yang, T., Wang, Z., Zhang, Y., Liu, S., and Shen, M. (2018). CircRNA CDRlas/miR-7 signals promote tumor growth of osteosarcoma with a potential therapeutic and diagnostic value. Cancer Manag. Res. 10, 4871-4880. doi: 10. 2147/CMAR.S178213

Xu, H., Guo, S., Li, W., and Yu, P. (2015). The circular RNA Cdrlas, via miR-7 and its targets, regulates insulin transcription and secretion in islet cells. Sci. Rep. 5:12453. doi: 10.1038/srep 12453

Xu, L., Zhang, M., Zheng, X., Yi, P., Lan, C., and Xu, M. (2017). The circular RNA ciRS-7 (Cdrlas) acts as a risk factor of hepatic microvascular invasion in hepatocellular carcinoma. J. Cancer Res. Clin. Oncol. 143, 17-27. doi: 10.1007/ s00432-016-2256-7

Yan, B., Zhang, W., Mao, X. W., and Jiang, L. Y. (2018). Circular RNA ciRS-7 correlates with advance disease and poor prognosis, and its down-regulation inhibits cells proliferation while induces cells apoptosis in non-small cell lung cancer. Eur. Rev. Med. Pharmacol. Sci. 22, 8712-8721. doi: 10.26355/eurrev_ 201812_16636

Yang, L., Bin, Z., Hui, S., Rong, L., You, B., Wu, P., et al. (2019). The Role of CDRlas in Proliferation and Differentiation of Human Umbilical CordDerived Mesenchymal Stem Cells. Stem Cells Int. 2019:2316834. doi: 10.1155/ $2019 / 2316834$ 
Yang, W., Gu, J., Wang, X., Wang, Y., Feng, M., Zhou, D., et al. (2019a). Inhibition of circular RNA CDR1as increases chemosensitivity of 5-FU-resistant BC cells through up-regulating miR-7. J. Cell Mol. Med. 23, 3166-3177. doi: 10.1111/ jcmm.14171

Yang, W., Yang, X., Wang, X., Gu, J., Zhou, D., Wang, Y., et al. (2019b). Silencing CDRlas enhances the sensitivity of breast cancer cells to drug resistance by acting as a miR-7 sponge to down-regulate REGgamma. J. Cell Mol. Med. 23, 4921-4932. doi: 10.1111/jcmm.14305

Yang, X., Li, S., Wu, Y., Ge, F., Chen, Y., and Xiong, Q. (2020). The circular RNA CDR1as regulate cell proliferation via TMED2 and TMED10. BMC Cancer 20:312. doi: 10.1186/s12885-020-06794-5

Yang, X., Xiong, Q., Wu, Y., Li, S., and Ge, F. (2017). Quantitative Proteomics Reveals the Regulatory Networks of Circular RNA CDRlas in Hepatocellular Carcinoma Cells. J. Proteome. Res. 16, 3891-3902. doi: 10.1021/acs.jproteome. 7 b00519

Yang, Z., Xie, L., Han, L., Qu, X., Yang, Y., Zhang, Y., et al. (2017). Circular RNAs: Regulators of Cancer-Related Signaling Pathways and Potential Diagnostic Biomarkers for Human Cancers. Theranostics 7, 3106-3117. doi: 10.7150/thno. 19016

Yao, W., Li, Y., Han, L., Ji, X., Pan, H., Liu, Y., et al. (2018). The CDR1as/miR7/TGFBR2 Axis Modulates EMT in Silica-Induced Pulmonary Fibrosis. Toxicol. Sci. 166, 465-478. doi: 10.1093/toxsci/kfy221

Yeldag, G., and Rice, A. (2018). Del Rio Hernandez A. Chemoresistance and the Self-Maintaining Tumor Microenvironment. Cancers 10:12. doi: 10.3390/ cancers10120471

Yu, L., Gong, X., Sun, L., Zhou, Q., Lu, B., and Zhu, L. (2016). The Circular RNA Cdrlas Act as an Oncogene in Hepatocellular Carcinoma through Targeting miR-7 Expression. PLoS One 11:e0158347. doi: 10.1371/journal.pone.015 8347

Yuan, W., Zhou, R., Wang, J., Han, J., Yang, X., Yu, H., et al. (2019). Circular RNA Cdrlas sensitizes bladder cancer to cisplatin by upregulating APAF1 expression through miR-1270 inhibition. Mol. Oncol. 13, 1559-1576. doi: 10.1002/18780261.12523

Zhang, J., Hu, H., Zhao, Y., and Zhao, Y. (2018). CDRlas is overexpressed in laryngeal squamous cell carcinoma to promote the tumour's progression via miR-7 signals. Cell Prolif. 51:e12521. doi: 10.1111/cpr.12521

Zhang, L., Li, Y., Liu, W., Li, H., and Zhu, Z. (2018). Analysis of the complex interaction of CDRlas-miRNA-protein and detection of its novel role in melanoma. Oncol. Lett. 16, 1219-1225. doi: 10.3892/ol.2018.8700

Zhang, S., Wang, W., Wu, X., and Zhou, X. (2020). Regulatory Roles of Circular RNAs in Coronary Artery Disease. Mol. Ther. Nucl. Acids 21, 172-179. doi: 10.1016/j.omtn.2020.05.024

Zhang, W., Zhang, C., Hu, C., Luo, C., Zhong, B., and Yu, X. (2020). Circular RNA-CDRlas acts as the sponge of microRNA-641 to promote osteoarthritis progression. J. Inflamm. 17:8. doi: 10.1186/s12950-020-0234-y

Zhang, X., Yang, D., and Wei, Y. (2018). Overexpressed CDRlas functions as an oncogene to promote the tumor progression via miR-7 in non-smallcell lung cancer. Onco Targets Ther. 11, 3979-3987. doi: 10.2147/OTT.S15 8316

Zhang, Y., Zhang, X. O., Chen, T., Xiang, J. F., Yin, Q. F., Xing, Y. H., et al. (2013). Circular intronic long noncoding RNAs. Mol. Cell 51, 792-806. doi: 10.1016/j.molcel.2013.08.017
Zhang, Z., Ren, L., Zhao, Q., Lu, G., Ren, M., Lu, X., et al. (2020). TRPC1 exacerbate metastasis in gastric cancer via ciRS-7/miR-135a-5p/TRPC1 axis. Biochem. Biophys. Res. Commun. 529, 85-90. doi: 10.1016/j.bbrc.2020.05.181

Zhao, F., Chen, T., and Jiang, N. (2020). CDR1as/miR-7/CKAP4 axis contributes to the pathogenesis of abdominal aortic aneurysm by regulating the proliferation and apoptosis of primary vascular smooth muscle cells. Exp. Ther. Med. 19, 3760-3766. doi: 10.3892/etm.2020.8622

Zhao, Y. H., Wang, Z., Zhang, N., Cui, T., and Zhang, Y. H. (2020). Effect of ciRS-7 expression on clear cell renal cell carcinoma progression. Chin. Med. J. 2020:867. doi: 10.1097/CM9.0000000000000867

Zhao, Y., Zheng, R., Chen, J., and Ning, D. (2020). CircRNA CDR1as/miR641/HOXA9 pathway regulated stemness contributes to cisplatin resistance in non-small cell lung cancer (NSCLC). Cancer Cell Int. 20:289. doi: 10.1186/ s12935-020-01390-w

Zhao, Z., Ji, M., Wang, Q., He, N., and Li, Y. (2019). Circular RNA Cdrlas Upregulates SCAI to Suppress Cisplatin Resistance in Ovarian Cancer via miR1270 Suppression. Mol. Ther. Nucleic Acids 18, 24-33. doi: 10.1016/j.omtn.2019. 07.012

Zheng, H. C. (2017). The molecular mechanisms of chemoresistance in cancers. Oncotarget 8, 59950-59964. doi: 10.18632/oncotarget.19048

Zhong, Q., Huang, J., Wei, J., and Wu, R. (2019). Circular RNA CDRlas sponges miR-7-5p to enhance E2F3 stability and promote the growth of nasopharyngeal carcinoma. Cancer Cell Int. 19:252. doi: 10.1186/s12935-019-0959-y

Zhou, R., Wu, Y., Wang, W., Su, W., Liu, Y., Wang, Y., et al. (2018). Circular RNAs (circRNAs) in cancer. Cancer Lett. 425, 134-142. doi: 10.1016/j.canlet.2018. 03.035

Zhou, X., Jiang, L., Fan, G., Yang, H., Wu, L., Huang, Y., et al. (2019). Role of the ciRS-7/miR-7 axis in the regulation of proliferation, apoptosis and inflammation of chondrocytes induced by IL-1beta. Int Immunophar. 71, 233240. doi: 10.1016/j.intimp.2019.03.037

Zhou, Y., Shen, L., Wang, Y. Z., and Zhou, C. C. (2020). The potential of ciRS-7 for predicting onset and prognosis of cervical cancer. Neoplasma 67, 312-322. doi: 10.4149/neo_2019_190415N334

Zou, Y., Zheng, S., Deng, X., Yang, A., Kong, Y., Kohansal, M., et al. (2020). Diagnostic and prognostic value of circular RNA CDR1as/ciRS-7 for solid tumours: A systematic review and meta-analysis. J. Cell. Mol. Med. 24, 95079517. doi: 10.1111/jcmm.15619

Zou, Y., Zheng, S., Deng, X., Yang, A., Xie, X., Tang, H., et al. (2019). The Role of Circular RNA CDRlas/ciRS-7 in Regulating Tumor Microenvironment: A Pan-Cancer Analysis. Biomolecules 9:429. doi: 10.3390/biom9090429

Conflict of Interest: The authors declare that the research was conducted in the absence of any commercial or financial relationships that could be construed as a potential conflict of interest.

Copyright (c) 2020 Jiang, Zeng, Shan, Wen, Li, Tan, Li and Wan. This is an openaccess article distributed under the terms of the Creative Commons Attribution License (CC BY). The use, distribution or reproduction in other forums is permitted, provided the original author(s) and the copyright owner(s) are credited and that the original publication in this journal is cited, in accordance with accepted academic practice. No use, distribution or reproduction is permitted which does not comply with these terms. 